\title{
Genomic structural and transcriptional variation of Oryctes rhinoceros nudivirus (OrNV) in Coconut Rhinoceros Beetle
}

Kayvan Etebari1*, Rhys Parry1, Marie Joy B. Beltran2 and Michael J. Furlong1

1- School of Biological Sciences, The University of Queensland, Brisbane, Australia.

2- National Crop Protection Centre, College of Agriculture and Food Science, University of the Philippines Los Baños College, Laguna 4031, the Philippines.

* Corresponding Author

Kayvan Etebari

School of Biological Sciences, Goddard Bldg. (No. 8)

The University of Queensland

St Lucia QLD 4072,

Australia

Tel: (+61 7) 33657086

Email: k.etebari@uq.edu.au 


\section{Abstract}

Oryctes rhinoceros nudivirus (OrNV) is a large circular double-stranded DNA virus which has been used as a biological control agent to suppress Coconut Rhinoceros Beetle (Oryctes rhinoceros) in Southeast Asia and the Pacific Islands. Recently a new wave of $O$. rhinoceros incursions in Oceania in previously non-infested areas is thought to be related to the presence of low virulence isolates of OrNV or virus tolerant haplotypes of beetles. In this study, chronically infected $O$. rhinoceros adults were field collected from the Philippines, Fiji, Papua New Guinea and the Solomon Islands. We extracted total RNA from these samples to investigate the global viral gene expression profiles and comparative genomic analysis of structural variations between the four different populations. Maximum likelihood phylogenic analysis indicated that OrNV strains from the Solomon Islands and the Philippines are closely related to while OrNV strains from PNG and Fiji formed a distinct adjacent clade. We detected several polymorphic sites with a frequency higher than $35 \%$ in 892 positions of the viral genome. The highest number of structural variants, including single nucleotide variants (SNV), insertion, deletion and non-synonymous mutations, were found in strains from Fiji and PNG when compared to complete recently sequenced Solomon Islands OrNV reference genome. Non-synonymous mutations were detected in several hypothetical proteins, and 15 nudivirus core genes such as $O r N V \_g p 034$ (DNA Helicase), lef-8, lef-4 and $v p 91$. For examination of the global gene expression profile of OrNV in chronically infected populations, we found limited evidence of variation between geographic populations. Only a few genes such as $O r N V \_g p 01$ (DNA polymerase B), OrNV_gp022 and $O r N V \_g p 107$ (Pif-3) were differentially expressed among different strains. Additionally, small RNA sequencing from the Solomon Islands population suggests that OrNV is targeted by the host RNA interference (RNAi) response with abundant $21 \mathrm{nt}$ small RNAs. Additionally, we identified a highly abundant putative $22 \mathrm{nt}$ miRNA from the $3^{\prime}$ of a pre-miRNA-like hairpin originating from OrNV-gp-098. These findings provide valuable resources for future studies to improve our understanding of the OrNV genetic variation. Some of these structural changes are specific to the geographic population and could be related to particular phenotypic characteristics of the strain, such as viral pathogenicity or transmissibility, and this requires further investigation.

Keywords: Oryctes rhinoceros nudivirus (OrNV), Coconut Rhinoceros Beetle, South Pacific Islands. Biological control, structural variation, viral transcriptome 


\section{Introduction}

Oryctes rhinoceros nudivirus (OrNV) (Genus Alphanudivirus) belongs to the diverse Nudiviridae family of rod-shaped viruses with large circular double-stranded DNA genomes which have a nuclear replication strategy in arthropod hosts. The typical pathology observed in nudivirus infected insects, and crustaceans suggest lethal infections in larvae and chronic disease in adults of various terrestrial and aquatic species $[1,2]$. The genome of OrNV encodes 130-140 genes [3, 4]. The coconut rhinoceros beetle (Oryctes rhinoceros), which is considered a globally invasive species [5] is a pest of palm trees and a severe threat to livelihoods in tropical Asia and the Pacific Islands. Oryctes rhinoceros is indigenous to South-East Asia, and OrNV was initially discovered and isolated from insects in Malaysia in 1963, providing a biological control agent for of $O$. rhinoceros that has been successfully used across the region for 50 years [6].

New incursions of the pest into previously $O$. rhinoceros-free countries and territories have been reported in recent years, beginning in Guam in 2007 and followed by Hawaii (2013), Solomon Islands (2015) and most recently Vanuatu and New Caledonia in 2019. It has been suggested that this resurgence and spread of the pest is related to OrNV resistant haplotypes, "CRB-G", which are identified by single nucleotide polymorphisms in the Cytochrome C Oxidase subunit I (CoxI) [7]. Since the release of OrNV into the Pacific, there have been some reports that indicate possible failures of the biocontrol agent including, repeated outbreaks of the beetle, the inconsistency of mortality caused by OrNV and lack of disease symptoms or low incidence of the virus in wild populations [8]. Accordingly, the presence of low virulence isolates of virus or tolerate haplotypes of beetles in different geographical regions has been proposed $[9,10]$. Several genomic variants of OrNV have been reported across the region based on endonuclease restriction analyses $[11,12]$. Still, the actual genetic diversity present within the wild type populations of OrNV remains unknown. Crawford and Zelazny (1990) detected the first viral structural variations four years after the initial release of different isolates of the virus in the Maldives. Using a restriction endonuclease digestion approach, they described some genomic alterations due to insertion and point mutation and also documented recombination between two of the strains of the virus released, suggesting rapid evolution of the virus [11].

Geographical populations of the virus may vary in virulence due to their genetic diversity. It has been reported that the failure of the virus to maintain control of $O$. rhinoceros in Malaysia may be due to low virulence of endemic OrNV strains [12-14]. The successful 
establishment of OrNV in O. rhinoceros populations is also critically dependent on virus transmissibility, and this too is likely to have a genetic basis. Different levels of transmission have been quantified for OrNV across the region. Still, too little information is available to understand the genetic basis of differences between the virulence and transmissibility of different geographical OrNV strains. Releasing several strains of the virus, as an essential component of pest management strategies, potentially intensifies the genetic diversity of OrNV in the region.

Previous studies have found widespread genomic variations in some large DNA viruses like baculoviruses [15, 16]. Many comparative analyses performed on baculovirus-host interaction models suggest that the differences in virulence and transmissibility involve multiple molecular pathways. Nonetheless, some mutations in key genes can be responsible for low transmissibility or virulence in a particular phenotype of the virus [17, 18]. A single wild type nuclear polyhedrosis virus (NPV) infected Panolis flammea caterpillar was found to contain 24 genotypic variants based on restriction fragment length polymorphism [19]. However, the fitness cost associated with genetic diversity in the population and whether or not this genetic diversity can be preserved in the population is unclear. Some non-synonymous mutations in core genes might have a significant impact on the virus fitness within different populations [16]. Although structural variants with low frequency can also be maintained within the population regardless of their fitness cost and can be a dominant genotype when suitable conditions arise [16].

The successful infection of the host gut tissue by OrNV particles is a crucial step that determines the success of the viral infection in O. rhinoceros. Many host and viral genes are involved in this critical event, as the midgut represents the first line of cellular defence against viral infection. A complex of proteins called per os infectivity factors (PIFs) mediate the binding and entry of virus particles into midgut epithelial cells. Undoubtedly, successful nudivirus infection involves a very complicated and coordinated expression of all viral genes. Any transcriptional variation or non-synonymous mutations of viral genes which disable the normal function of these proteins could reduce virus virulence and interrupt its vertical or horizontal transmission.

Unfortunately, lack of information on the global gene expression pattern of OrNV in persistently (or chronically) infected insect hosts or even in cultured insect cells prevents us from having a clear view of host-pathogen interactions at the viral gene transcriptional level. We recently detected an extremely high prevalence of viral infection in $O$. rhinoceros adult 
specimens across the region, even in those host haplotypes (CRB-G) which believed to be resistance to OrNV (unpublished data). Here we present the first genome-wide study of the genomic diversity of OrNV strains across the Pacific. This study generated a compendium of OrNV structural variants and gene expression profiles in different geographical strains which infect the different mitochondrial lineages (CRB-G, CRB-S and CRB-PNG) of O. rhinoceros. Furthermore, some of these structural changes are specific to different geographic populations and could be related to particular phenotypic characteristics of the strain, such as viral pathogenicity or transmissibility.

\section{Methods}

\subsection{Sample collection and processing}

Adult female Coconut Rhinoceros Beetles (O. rhinoceros) were collected from the Philippines (Los Baños) and three different South Pacific countries, Fiji (Sigatoka, Viti Levu), Papua New Guinea (Kimbe, New Britain) and Solomon Islands (Honiara, Guadalcanal) using traps baited with aggregation pheromone (Oryctalure, P046-Lure, ChemTica Internacional, S. A., Heredia Costa Rica) between June-October 2019.

Individual female insects were disinfected by soaking in $75 \%$ Ethanol for 30s and then rinsing in phosphate-buffered saline (PBS) before their gut tissues were dissected, removed and preserved in an RNA stabilisation reagent (RNAlater ${ }^{\circledR}$, QIAGEN). For each individual, some fat body and small pieces of gut tissues were also preserved separately in $95 \%$ ethanol. Preserved specimens were shipped to the University of Queensland, Brisbane, Australia for further analysis. The preserved gut tissues were kept at $-80{ }^{\circ} \mathrm{C}$ upon arrival. For examination of virus infection status and mitochondrial haplotype, DNA was extracted from fat bodies and gut fragments using Qiagen Blood and Tissue DNA extraction kit according to manufacturers instruction.

Presence of the OrNV was confirmed by successful amplification of a 945 bp product using the OrV15 primers that target OrNV-gp083 gene [20] and by Sanger sequencing of each PCR product. For confirmation of the mitochondrial haplotype, a small fragment of CoxI gene was amplified in these individuals with the primers LCO1490 and HCO2198 [21]. PCR products were sequenced bi-directionally using an ABI3730 Genetic Analyser (Applied Biosystems) at Macrogen Inc. Seoul, South Korea. 
The gut from OrNV positive individuals was further dissected and transferred to Qiazol lysis reagent for RNA extraction according to manufacturer's instructions (QIAGEN; Cat No.: 79306). The RNA samples were treated with DNase I for $1 \mathrm{hr}$ at $37^{\circ} \mathrm{C}$ and then their concentrations were measured using a spectrophotometer and integrity was ensured through analysis of RNA on a $1 \%(\mathrm{w} / \mathrm{v})$ agarose gel. After checking the RNA quality, total RNA samples were submitted to the Novogene Genomics Singapore Pte Ltd for RNA sequencing (RNA-seq). The PCR-based cDNA libraries were prepared using NEBNext ${ }^{\circledR}$ Ultra $^{\mathrm{TM}}$ RNA Library Prep Kit and were sequenced using Novaseq6000 (PE150) technology with the insert size between 250-300 bp.

\subsection{Bioinformatic analysis}

The CLC Genomics Workbench version 12.0.1 was used for bioinformatics analyses. All libraries were trimmed from any vector or adapter sequences remaining. Low quality reads (quality score below 0.05) and reads with more than two ambiguous nucleotides were discarded. For identification of the virus consensus from each sample, clean reads mapped to a recently reported Oryctes rhinoceros nudivirus complete genome sequence (MN623374) [4]. We implemented strict mapping criteria (mismatch, insertion and deletion costs: 2: 3: 3 respectively). The minimum similarity and length fraction of 0.9 between a mapped segment, and the reference was allowed in mapping criteria. We also permitted minimum 5 reads as low coverage threshold and inserted $\mathrm{N}$ as ambiguity symbols for handling the low coverage region. The quality score for each nucleotide has been used to resolve any conflict. For production of multiple sequence alignments, we used MUltiple Sequence Comparison by Log-Expectation (MUSCLE v3.8.425) to compare OrNV whole-genome consensus sequence from different libraries.

\subsubsection{Structural variant and phylogenetic analysis}

To detect structural modifications among different OrNV infected $O$. rhinoceros populations, CLC Genomic Workbench low-frequency variant detection tool, which is based on a neighbourhood quality standard (NQS) algorithm was used. Variants such as single nucleotide variant (SNV) multiple nucleotide variant (MNV), insertion, deletion and amino acid modification were then called based on neighbourhood radius (5), a maximum number of gaps and mismatches (2), the minimum average quality of surrounding bases (15), and 
minimum quality of the central base (20). We also used a minimum coverage filter of 10 and a minimum variant frequency of $35 \%$ to exclude sequencing artefacts. The final alignments for downstream analysis were OrNV 11x126,183nt and for the partial CoxI 11x621nt. For the construction of the maximum-likelihood phylogenetic tree of both host mitochondrial DNA and OrNV, we selected a nucleotide substitution model based on the consensus outcome of hierarchical likelihood ratio test (hLRT), Bayesian information criterion (BIC), Akaike information criterion (AIC). For the OrNV phylogenetic inference the General Time Reversible $(\mathrm{GTR})+\mathrm{G}$ rate variation (4 categories), $+\mathrm{T}$ topology variation was selected from all tests. For the mitochondrial CoxI the Hasegawa-Kishino-Yano (HKY) substitution model with $+\mathrm{T}$ topology variation was selected. The maximum-likelihood phylogeny was then constructed using CLC Genomics Workbench with a Neighbour Joining starting tree construction method under the nucleotide-substitution models mentioned above with 1000 bootstraps. Resultant trees were exported and visualised using FigTree version 1.4 (A. Rambaut; http://tree.bio.ed.ac.uk/software/figtree/).

\subsubsection{Viral gene expression profiling}

For examination of OrNV gene expression profiles in different $O$. rhinoceros populations, clean reads were mapped to the OrNV genome using the RNA Sequencing Tool on CLC genomics workbench. Expression values for all OrNV genes were calculated as total mapped read count and then normalised to reads per kilobase of transcript per million mapped viral reads (RPKM). The most highly expressed OrNV genes in O. rhinoceros gut tissue in different populations were identified and ranked. The viral gene expression profile was created based on calculated RPKM values. For comparison of viral gene expression analysis among different OrNV infected $O$. rhinoceros populations, hierarchical clustering analysis with Euclidean distance metric on the log2-transformed RPKM value (normalised expression value) were performed. Differential expression profiles between the four different populations were modelled using a separate Generalised Linear Model (GLM). The use of the GLM formalism allows the fitting of curves to expression values without assuming that the error on the values is normally distributed. The Wald test was also used to compare each sample against its control group to test whether a given coefficient is non-zero. We considered genes with more than 2fold changes and a false discovery rate (FDR) of less than 0.05 as significantly modulated viral genes. For further functional annotation of OrNV hypothetical proteins, we search for a 
conserved domain through IntrProScan database [22]. We also used EggNOG version 4.5 to run pairwise orthology prediction on Cluster of Orthologous Groups [23].

\subsubsection{Viral derived small RNA analysis and OrNV miRNA prediction}

For analysis of the host RNAi response to OrNV, a small RNA library was generated from a virus-infected individual from the Solomon Islands using the NEBNext ${ }^{\circledR}$ Multiplex Small RNA Library Prep Kit for Illumina ${ }^{\circledR}$ at the Novogene Genomics Singapore Pte Ltd. The purified cDNA libraries were sequenced on Novaseq 6000 (SE50) and raw sequencing reads were obtained using Illuminas Sequencing Control Studio software. Raw data were stripped of adapters and reads with quality score above 0.05 , and less than two ambiguous nucleotides were retained. Reads without 3' adapters and also reads with less than 16 nt were discarded. The clean reads mapped to the recently sequenced OrNV genome (MN623374). For examination of potential viral derived miRNAs of OrNV we used miRDeep v2.0.0.8 [24] hosted on the Mississippi Galaxy instance (https://mississippi.sorbonne-universite.fr/). We screened the genome consensus of all OrNV geographical strains for potential miRNA binding sites with miRanda algorithm [25]. This tool considers matching along the entire miRNA sequence, but we ran the program in strict mode, which demands strict 5' seed pairing.

\section{Results and Discussion}

\subsection{Evidence of co-evolution of OrNV strains with their $O$. rhinoceros hosts}

In this study $O$. rhinoceros adults were collected from the Philippines in their native region and from Fiji (Sigatoka, Viti Levu), Papua New Guinea (Kimbe, New Britain) and the recently invaded Solomon Islands (Honiara, Guadalcanal) in non-native regions in the South Pacific Islands (Figure 1A). The total RNA from adults $O$. rhinoceros infected with wild type OrNV were sequenced, and in total, 42,678,016 Illumina paired-end reads mapped to the OrNV genome. The percentage of reads mapped to the virus genome in each library was between $0.53-18.92 \%$ of the total reads (Table 1 ). We used the recently sequenced Solomon Islands strain of OrNV genome (MN623374) as a reference in this study [4], and the viral genome consensus sequences have been generated for each library (Figure S1).

Maximum likelihood phylogeny analysis of four different wild type populations of OrNV based on their complete genome sequence consensus generated two major clades. These 
data show that samples from Solomon Islands and Philippines are closely related to each other while samples from PNG and Fiji are separated from them and located in another clade (Figure 1). The phylogenic analysis of field-collected $O$. rhinoceros based on the host partial CoxI gene revealed three major mitochondrial lineages, CRB-G, CRB-S and CRB-PNG. All $O$. rhinoceros individuals collected from PNG (Kimbe, New Britain) and Fiji belonged to the mitochondrial lineages CRB-PNG and CRB-S, respectively. The individuals from the Philippines and the recently invaded Solomon Islands belonged to the CRB-G clade (Figure 1C). The virus strains from the Solomon Islands and the Philippines also belonged to one major clade (Figure 1B). Harmonious topologies of both host and OrNV strains provide evidence of ongoing co-evolution of OrNV strains with their O. rhinoceros hosts.

Previously Marshall et. al., (2017) reported that the natural infection rate of OrNV ranges from $45.2 \%$ to $64.5 \%$ in $O$. rhinoceros from a wide range of countries in Southeast Asia and Pacific. However, they couldn't detect the presence of the OrNV in specimens from the Solomon Islands, Philippines and Oahu which were believed to be the CRB-G haplotype. The source of the virus found in our 2019 collection from the Solomon Islands is not known [4]; it could have been introduced deliberately as a biological control agent or along with an incursion of infected beetles from neighbouring islands (CRB-PNG from PNG). However, due to high the high level of similarity and lack of any evidence that OrNV (Philippines strain) was introduced into the country as a biological control agent, our work suggests that the OrNV came to the Solomon Islands with an incursion of infected CRB-G that originated in Southeast Asia.

\subsection{Whole genome structural variant analysis of OrNV strains}

We detected several SNPs with a frequency higher than $35 \%$ in 892 positions of the 125917 bp viral genome sequence, which found in pair-end reads positioned in both orientations of the genome. In samples collected from the Solomon Islands, 87-141 variants were identified in which deletion is their major variation when compared to the newly sequenced genome reference (MN623374). The maximum number of structural variants such as single nucleotide variants (SNV), insertion, deletion and the number of variants in amino acid (non-synonymous mutations) were found in individuals from Fiji and PNG (Figure 2). We detected 12-17 proteins with amino acid modifications in samples collected from the Solomon Islands while this number reached to 61-63 proteins in samples from Fiji and PNG. OrNV in 
the Philippines showed more similarity to OrNV in individuals from Solomon Islands based on the number of identified SNV and non-synonymous modifications. Some of the structural variants we identified could be involved in differences in viral transmission and virulence.

Structural variants have been found in both coding and non-coding regions of the OrNV genome. In all individuals from Fiji, Philippines and PNG, the number of variants in coding regions is higher than the number in the non-coding part of their genomes (Figure 3). Generally, mutations in coding regions are likely to have a significant impact on the genome, whereas, in non-coding regions, such mutations are more likely to be neutral. However, certain non-coding regions of baculoviruses could be important components of the viral genomes. [17].

Non-synonymous mutations which do result in amino acid modification and could potentially interrupt protein function were detected in 15 nudivirus core genes and several hypothetical proteins (Table 2 and Table S1). We found up to four amino acid modifications in DNA Helicase (QHG11272.1) among individuals from different populations. A nonsynonymous mutation (c.5C>T) in $\operatorname{OrNV}$ gp034 caused an amino acid modification in DNA helicase in and individual sample from Solomon Islands. This amino acid substitution from Threonine to Methionine can also change the biochemical properties (polarity changed from neutral polar to non-polar) of this critical protein, and therefore it might affect protein function and possibly virus performance. Other non-synonymous mutations in this gene do not change the polarity of the protein. One of these amino acid changes (p.Leu211Phe) is specific to samples isolated from Fiji. Previous studies in baculoviruses showed that this gene is associated in host range expansion. Mutations in the genes involved in DNA replication might affect the rate of virion production and thus might alter the spread of the infection within the host [17]. DNA Helicase is one of the most rapidly evolving genes in Drosophila innubila nudivirus (DiNV) and Kallithea virus and may be important for adaptation to a new host system [26, 27].

We identified non-synonymous mutations in several genes involved in transcription, including three subunits of the RNA polymerase (lef-4, lef-8 and lef-9) (Table 2 and Table S1). Interestingly, a similar event has been reported from AcMNPV [16] and Spodoptera exigua multiple nucleopolyhedrovirus (SeMNPV) [17]. Here, amino acid variants in lef-9 do not change protein polarity. Still, non-synonymous mutations in lef-8 and lef-4 had an impact on biochemical properties of the protein in some individuals from Fiji and PNG. OrNV_gp64 which encode lef-8 protein is also overexpressed in OrNV collected from Fiji (Fold change 4.38, FDR: 0.048) and the lowest expression value was detected in individuals from the Philippines. lef-4 is an RNA capping enzyme and was originally identified as being essential 
for late transcription. A deletion mutant in the BmNPV lef-8 homolog (Bm39) was not viable, and inactivation of BmNPV lef-8 prevented virus replication [28, 29]. Theze et al (2014) also suggested that mutations in the subunits of the viral RNA polymerase, lef- 9 and $p 47$ ORFs are associated with vertical transmission and pathogenicity. We did not detect any mutations in OrNV_gp020, which encodes 47 protein, in individuals the sequenced from across the pacific suggesting that this gene is essential for OrNV replication. However, it is challenging to definitively link specific mutations to virus performance because virulence and virus performance is derived from the combination of the genetic material present at the wholegenome scale.

Genes involved in primary infection encoding essential components of the per os infectivity complex such as pif-1, pif-2, pif-3 also have some non-synonymous mutations in their sequences across the different geographical population of OrNV (Table 2 and Table S1). The products of pif genes, PIF proteins, which are conserved across the baculoviruses and nudiviruses, bind to insect midgut cells and are essential for infection. We only detected one amino acid modification in pif-3 and pif- 2 in one of the samples collected from the Philippines. There is a conserved amino acid change (p.Val184Ile) in pif- 1 of all individuals from the PNG population, but it has no impact on protein polarity. Chateigner et al (2015) suggested that the presence of different variants of the PIF proteins in the AcMNPV might allow the binding of these proteins to a wider range of host cell, which could be advantageous for establishment of viral infection.

OrNV_gp126 which encodes p74 protein is another gene with a role in per os infectivity, and we detected amino acid changes in all individuals. This protein is critical in host-pathogen interactions in the insect midgut, and its deletion prevents the recombinant virus transmission in insects, but the mutation does not affect replication in cultured cells [30]. Two of the amino acid changes were specific to the Fiji population and altered protein polarity.

The very late factors (VLF) appear to be a structural, conserved protein that is present in both baculoviruses (BV and ODV) and nudiviruses and which is required for the production of nucleocapsids. Several deletion/insertions of serine (p349^350) and glutamine (p644^645) have been detected in $v l f-1$ in all the OrNV samples; this could be due to a sequencing error (homopolymer stretches) or the presence of actual mutation sites (Table 2 and Table S1). We also detected two mutation sites in $O r N V \_g p 106$, which encodes VP91 protein. One of those mutations (p.Glu564Asp) is specific to OrNV from the Solomon Islands and the Philippines, and another (p.Ala117Thr) is specific to OrNV from Fiji. Changing alanine to threonine also 
alters protein polarity from non-polar to polar, and it could cause critical conformational changes affecting protein structure. Theze et al (2014) showed that some variants of $v p 91$ in SeMNPV could be associated with pathogenicity [17].

\subsection{Limited evidence of OrNV gene expression variation between chronically infected populations.}

The principal component analysis of OrNV samples from different countries based on overall viral gene expression profiles can differentiate each of the OrNV populations. Two samples from Fiji had similar profiles, and their expression pattern is slightly different from the other populations (Figure 4). Two individuals from the Solomon Islands and Philippines are located outside of their population cluster, probably due to different stages of infection, specific gene expression patterns and low viral reads in those libraries (Figure 4 and Table 1). However, we performed library size normalisation using the TMM (trimmed mean of $\mathrm{M}$ values) method and library sizes are then used as part of the pre-sample normalisation. To get a better view of gene expression profile in each sample, we ranked the genes based on their RPKM values and filtered the most highly and poorly expressed gene in each library.

OrNV_gp41 is the most highly expressed gene with an unknown function in all individual libraries. This 342 bp nudivirus specific gene shows significant similarity to GrBNV_gp95 and does not have a known homolog in Baculoviruses. Further sequence analysis using InterProScan and GO database showed that some parts of $\mathrm{OrNV} g p 41$ gene product are integral components of the hydrophobic region of the membrane (GO:0016021). This virus fragment also integrated into the brown planthopper (Nilaparvata lugens) genome [31]. Recently, it has been identified as endogenous nudivirus-like viruses of parasitoid wasps in $\mathrm{CcBV}$ (Cotesia congregate Bracovirus), $\mathrm{CiBV}$ (Chelonus inanitus Bracovirus) and its expansion into a gene family in a wasp genome also reported from Venturia canescens viruslike particles (VcVLP) [32].

OrNV_gp022 (GrBNV_gp72-like) is a member of alphanudivirus core genes with unknown function, which is in the top 10 highly expressed genes in all OrNV populations. However, its normalised expression value in the Fiji population is significantly lower than in other samples (Figure 5 and Table S2). Other genes among the top 10 most highly expressed

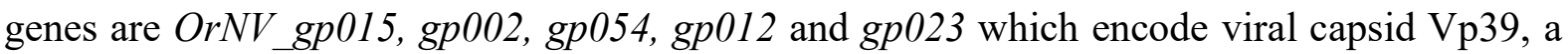


trypsin-like serine protease, glycoprotein gp83-like, odv-e66 and guanylate kinase-like proteins, respectively.

Trypsin-like serine proteases are non-structural proteins with a conserved domain (c121584) which are found in several RNA and DNA viruses and cellular organisms [33]. These proteins are essential for virus maturation [34], and they are involved in proteolysis through serine-type endopeptidase activity and post-translational modifications of these enzymes play a crucial role in virus replication [35]. Previously, it has been shown that trypsin-serine proteases have dN/dS above 0.5 between DiNV and OrNV, which indicate that this gene is under purifying selection $(\mathrm{dN} / \mathrm{dS}<1)$ and unconstrained evolution or putative adaptation $(\mathrm{dN} / \mathrm{dS}>0.5)$ [26]. However, we did not detect any amino acid modification in this gene within the different OrNV populations.

Recently, it has been experimentally demonstrated that a nudivirus encoded protein gp83 inhibits the Toll signalling pathway in the fruit fly. Drosophila melanogaster Kallithea virus (KV), which is a new member of family Nudiviridae and DiNV suppress host immune responses by regulating NF-kB transcription factors [36]. This immunosuppressive activity of gp83 against $D$. melanogaster $\mathrm{NF-kB}$ signalling is conserved and probably gp 83 shows similar activity in other members of Nudiviridae. This gene is the second most highly expressed gene in samples collected from Fiji, and its normalised expression value is slightly higher than in other populations (Figure 5).

ODV-E66 protein which has a Chondroitin AC/ alginate lyase (IPR008929) domain, potentially involves viral envelope formation and facilitates viral protein movement during infection by degrading larval peritrophic membrane [37]. This protein has been reported from baculoviruses and other nudiviruses (such as $\mathrm{HgNV}$ and Penaeus monodon nudivirus), and it has been suggested that ODV-E66 is a per os infectivity factor [2,38]. Although odv-e66 is not a top 20 most highly expressed gene in AcMNPV, its expression pattern has a strong negative correlation between Trichoplusia $n i$ midgut and $T$. $n i$ cell line (Tnms42). Overexpression in midgut tissue confirms their responsibility for the trafficking of viral proteins during infection [18].

OrNV_gp107 which encodes pif-3 is highly expressed in OrNV strains from the Philippines, and its expression is 3.56 times higher than in samples collected from Fiji (Figure 5C). Although expression of pif genes are necessary for successful infection of gut tissue, it has been shown that over-expression pif- 1 in Spodoptera frugiperda NPV is unfavourable for 
viral population fitness and can cause more mutation in this gene and develop viral genotypes with lack of pif-1 expressing capabilities [39]. There are several pif genes in OrNV which encode essential components of envelope proteins and contain an N-terminal hydrophobic sequence in combination with several adjacent positively charged amino acids.

Most of the OrNV poorly expressed genes encode hypothetical proteins, and we don't have enough information about their potential role in the host-pathogen interaction. OrNV gp99 which encodes Ac120-like protein is one the most poorly expressed genes in OrNV but their expression was significantly upregulated in samples collected from Fiji and almost undetectable in Philippines and Solomon Islands populations (Figure 5, Table S2). We recently found six nucleotides substitution in $\operatorname{OrNV} g p 99$ when we compared the Solomon Islands strain with Ma07 from Malaysia, and several amino acid modifications were detected among samples analysed in the current study (Table S1). Homologs of this gene have been found in other baculoviruses and nudiviruses, and it is likely to be nonessential, as an insertion/deletion mutation of this gene in BmNPV (Bm98) had no apparent effect on infectivity [40].

$O r N V \_g p 01$ which encodes DNA polymerase B has low expression value in almost all libraries (Rank 97 out of 130 genes), but it is overexpressed (Fold change 2.7, FDR: 0.002) in samples collected from Fiji (Figure 5C).

\subsection{OrNV is targeted by the host RNAi response and encodes a putative miRNA}

During virus infection in arthropods, two standard small RNA classes; the microRNAs (miRNAs) and small-interfering RNAs (siRNAs) contribute to the antiviral response, promote viral tolerance and in some cases, clearance of virus infections (Reviewed by [41, 42]). During DNA virus infection, the host riboendonuclease III enzyme Dicer-2 cleaves double-stranded RNA (dsRNA) into virus-derived small interfering RNAs (vsiRNAs) 20-22 nt in length [43, 44]. These vsiRNAs are loaded into the RNA-induced silencing complex (RISC), where they target RNA molecules through complementarity, reduce DNA virus gene transcription and ultimately virus replication. The RNAi response in DNA virus infection is subtly different to the RNAi response to RNA virus infections as dsDNA viruses do not require an RNA genome intermediate for replication. As such, the biogenesis of vsiRNAs are abundantly generated from the bi-directional transcription of overlapping gene regions [43] and also secondary RNA structures [45]. Some secondary structures of viral RNA are processed by the canonical host 
miRNA machinery and appear to be functional miRNAs [46]. These miRNAs influence virus latency in the case of Heliothis zea Nudivirus [47], but can also autoregulate transcription of DNA virus genes [48].

To our knowledge, only one study has examined the virus RNAi response in beetles with virally derived siRNAs demonstrated in the red flour beetle (Tribolium castaneum) infected with the single-stranded positive-sense RNA Tribolium castaneum iflavirus (Iflavirdae) [49]. To examine the repertoire of small RNA responses to OrNV, the small RNA fraction (16-32nt) of a persistently infected sample from the Solomon Islands was subject to small RNA sequencing and mapped against the OrNV genome. We examined both the size distribution of the viral derived RNA fragments as well as "hot-spot" genomic locations. After quality and adapter trimming of the small RNA sequencing library (Figure 6A) 563,774 of the 11,121,239 small RNA 16-32nt reads (5.07\%) could be mapped to both orientations of the OrNV genome. Examination of the size profile of these mapped small RNA reads (Figure 6B) suggested that the majority $(57.7 \%)$ correspond to the $21 \mathrm{nt}$ prototypical Dicer-2 vsiRNAs. After examination of the strand origin of the viral derived small RNA read population, reads originated from both the forward (52.7\%) and reverse orientation (47.3\%) of the genome more or less evenly. The genomic regions responsible for the high generation of vsRNAs in the OrNV genome suggested that the biogenesis of vsRNAs from the OrNV were unevenly targeted throughout the OrNV genome with "hot spot" regions as previously demonstrated in other DNA-insect RNAi responses [43,44]. This is evidenced by coverage on both forward and reverse sequences similar to a pattern of "mirroring" of vsiRNA between genome position (Figure 6C).

One portion of high coverage with vsRNA reads without a corresponding region of high coverage originating from overlapping negative genome orientation at approximately $85.5 \mathrm{~kb}$ of the OrNV genome (Figure 6C). As a previous report from Drosophila infected with Kallithea Virus (Nudivirdae), a close relative of OrNV, suggests that Kallithea Virus encodes a miRNA [50] we examined the possibility that OrNV also potentially produces a miRNA. For this small RNA reads originating from the OrNV genome were analysed by the miRNA prediction tool mirDeep2 [24]. A single putative $22 \mathrm{nt}$ miRNA (UUGGAUUUUGUCCGUCGACGAG) from the $3^{\prime}$ of a pre-miRNA-like hairpin originating from the genomic location corresponding to a high peak of small RNAs (85464-85520 on OrNV-gp-098) (Figure $6 \mathrm{C}-\mathrm{D}$ ). The mature sequence and isomir variants of this putative miRNA (herein described as OrNV-miR-1-3p) were highly abundant in the whole sRNA library and in total considering 2 
nucleotide upstream and 5 nucleotide downstream amounted to almost $2.6 \%$ of all OrNV reads with $(14,595$ reads).

Additionally, the mature OrNV-miRNA-1 was the single most abundant sRNA sequence of the OrNV derived small RNAs with the exact 22nt accounting for 4832 reads $(0.8 \%)$. There were only 65 reads in total associated with -5p RNA "star" sequence (OrNVmiR-1-5p: CCGUCGACGCGCAACGUUCGA) (Figure 6E).

Upon further examination of the miRNA, we found that there was no apparent homology to other coleopteran or arthropod miRNAs as per similarity analysis to miRNAs annotated on miRBase (Release 22.1: October 2018) [51] and the seed region displays no obvious similarity to known miRNAs. Unfortunately, as the genome of $O$. rhinoceros is not currently available, we are not able to conduct a preliminary analysis to potential OrNV-miR1/host gene mRNA interactions however miRNA could alternatively regulate virus gene expression of OrNV itself [46]. We identified several potential binding sites on the genome of different geographical strains of OrNV (Supplementary Figure S2). Although 49 potential binding sites are universal among all strains, there are some binding sites exclusively identified for Fiji, PNG and the Philippines, which are not detected in other populations (Supplementary Figure S2). While numbers of OrNV-miR-1 are far lower than the $22 \mathrm{nt}$ miRNA reported from Kallithea Virus, which represented $>35 \%$ of all reads [50], given the evidence that other large dsDNA viruses with nuclear replication strategies also encode miRNAs and the absence of overlapping transcription or high -5p or "star" reads indicates that OrNV-miR-1 is a promising candidate for future laboratory studies in the contribution to long term chronic OrNV persistence in infected $O$. rhinoceros beetles.

\section{Conclusion}

We identified a high number of polymorphic sites among several geographical strains of OrNV, but potentially only a few of these variations in the genome are involved in functional changes. We found some structural variations in OrNV core genes such as OrNV_gp034 (DNA Helicase), lef-8, lef-4 and $v p 91$, which can potentially alter their typical function. These findings provide valuable resources for future studies to improve our understanding of the OrNV genetic variation in different geographic regions and their potential link to virus pathogenicity. However, further genomic analyses of OrNV strains in combination with the characterisation of its effects on the host across multiple populations is necessary to 
comprehend if such structural or transcriptional variation is responsible for different levels of effectiveness of OrNV as a biological control agent in different countries.

\section{Data Availability}

Deep sequencing data have been deposited in NCBI's Gene Expression Omnibus and are accessible through GEO series accession numbers GSExxxx and GSExxxx (pending accession numbers).

\section{Author Contribution}

Kayvan Etebari: Conceptualisation, Methodology, Investigation, Formal analysis, Data curation, Visualisation, Writing- Original draft preparation, Funding acquisition

Rhys Parry: Data curation, Formal analysis, Visualisation, Writing- Original draft preparation Marie Joy B. Beltran: Resources

Michael J. Furlong: Conceptualisation, Writing- Reviewing and Editing, Funding acquisition, Project administration, Supervision

\section{Acknowledgements}

This project was supported by the Australian Centre for International Agricultural Research funding (HORT/2016/185) and the University of Queensland (UQECR2057321). We would also like to thank Apenisa Sailo (Ministry of Agriculture, Fiji), Helen Tsatsia (Ministry of Agriculture and Livestock, Solomon Islands) and colleagues from Papua New Guinea Oil Palm Research Association, Dami research station for their assistance in collecting and providing the insect specimens. 
bioRxiv preprint doi: https://doi.org/10.1101/2020.05.27.119867; this version posted May 27,2020 . The copyright holder for this preprint (which was not certified by peer review) is the author/funder, who has granted bioRxiv a license to display the preprint in perpetuity. It is made available under aCC-BY-NC-ND 4.0 International license.

Table 1. The distribution of viral reads among different RNAseq libraries

\begin{tabular}{llcccc}
\hline Population & Sample ID & $\begin{array}{c}\text { Reads mapped to } \\
\text { viral genome }\end{array}$ & $\begin{array}{c}\text { Viral reads in } \\
\text { library (\%) }\end{array}$ & $\begin{array}{c}\text { Gene } \\
(\%)\end{array}$ & $\begin{array}{c}\text { Intergenic } \\
(\boldsymbol{\%})\end{array}$ \\
\hline Fiji & F5 & 465,294 & 0.91 & 94.58 & 5.42 \\
Philippines & F6 & $2,498,061$ & 6.34 & 93.63 & 6.37 \\
& PH2 & $8,188,367$ & 18.92 & 94.08 & 5.92 \\
& PH4 & $6,129,400$ & 13.22 & 93.69 & 6.31 \\
PNG & PH8 & 525,590 & 0.93 & 92.88 & 7.12 \\
& PNG2 & $1,361,190$ & 3.21 & 93.31 & 6.69 \\
& PNG4 & $5,421,019$ & 12.65 & 93.58 & 6.42 \\
& PNG5 & $4,919,054$ & 11.87 & 94.1 & 5.9 \\
& PNG6 & $5,516,617$ & 13.62 & 93.28 & 6.72 \\
& SI24 & $7,406,766$ & 14.33 & 94.04 & 5.96 \\
& SI30 & 246,658 & 0.54 & 91.54 & 8.46 \\
\hline
\end{tabular}


Table 2. Number of amino acid modifications in OrNV core gene products.

\begin{tabular}{|c|c|c|c|c|c|c|c|c|c|c|c|c|c|c|}
\hline Gene ID & Gene & Gene Product & Protein NCBI ID & FIJI 1 & FIJI 2 & PHI 1 & PHI 2 & PHI 3 & PNG 1 & PNG 2 & PNG 3 & PNG 4 & SI 1 & SI 2 \\
\hline \multicolumn{15}{|c|}{ DNA replication: } \\
\hline OrNV_gp001 & dnapol & DNA polymerase B & QHG11240.1 & 2 & 2 & 4 & 2 & 2 & 2 & 2 & 2 & 2 & 2 & - \\
\hline OrNV_gp034 & helicase & DNA helicase & QHG11272.1 & 4 & 4 & 3 & 1 & 2 & 3 & 3 & 3 & 3 & - & - \\
\hline \multicolumn{15}{|l|}{ Transcription: } \\
\hline OrNV_gp020 & $p 47$ & p47 protein & QHG11259.1 & - & - & - & - & - & - & - & - & - & - & - \\
\hline OrNV_gp064 & lef-8 & late expression factor 8 & QHG11301.1 & - & - & 1 & - & - & 1 & 1 & - & - & - & - \\
\hline OrNV_gp096 & lef-9 & late expression factor 9 & QHG11328.1 & 2 & 2 & 1 & 1 & 1 & 2 & 2 & 2 & 2 & - & - \\
\hline OrNV_gp042 & lef-4 & late expression factor 4 & QHG11280.1 & 1 & 1 & - & - & - & - & - & - & - & - & - \\
\hline OrNV_gp052 & lef-5 & late expression factor 5 & QHG11289.1 & - & - & - & - & - & - & - & - & - & - & - \\
\hline OrNV_gp059 & lef-3 & late expression factor 3 & QHG11296.1 & - & - & - & - & - & - & - & - & - & - & - \\
\hline \multicolumn{15}{|c|}{ Envelope component: } \\
\hline OrNV_gp017 & pif-2 & per os infectivity 2 & QHG11256.1 & - & - & - & - & 1 & - & - & - & - & - & - \\
\hline OrNV_gp060 & pif-1 & per os infectivity 1 & QHG11297.1 & - & - & 2 & - & - & 1 & 1 & 1 & 1 & - & - \\
\hline OrNV_gp107 & pif-3 & per os infectivity 3 & QHG11339.1 & - & - & 1 & - & - & - & - & - & - & - & - \\
\hline OrNV_gp033 & pif-4 & $19 \mathrm{kDa}$ protein & QHG11271.1 & - & - & - & - & - & - & - & - & - & - & - \\
\hline OrNV_gp126 & $p 74$ & p74 protein & QHG11358.1 & 3 & 3 & 1 & 1 & 2 & 1 & 1 & 1 & 1 & 1 & 1 \\
\hline OrNV_gp004 & $a c 81$ & Ac81-like protein & QHG11243.1 & - & - & 1 & - & 1 & - & - & - & - & - & - \\
\hline OrNV_gp113 & p33 & Ac92-like protein & QHG11345.1 & - & - & - & - & - & - & - & - & - & - & - \\
\hline \multicolumn{15}{|c|}{ Capsid component: } \\
\hline OrNV_gp087 & $38 K$ & $38 \mathrm{~K}$ protein & QHG11320.1 & - & - & - & - & - & - & - & - & - & - & - \\
\hline OrNV_gp072 & ac68 & Ac68-like protein & QHG11307.1 & - & - & - & - & - & - & - & - & - & - & - \\
\hline OrNV_gp030 & $v \mid f-1$ & very late expression factor 1 & QHG11269.1 & 2 & 2 & 2 & 2 & 1 & 2 & 2 & 2 & 2 & - & - \\
\hline OrNV_gp022 & $g p 72$ & GrBNV_gp72-like protein & QHG11261.1 & 1 & 1 & - & - & - & 1 & 1 & 1 & 1 & - & - \\
\hline OrNV_gp015 & $v p 39$ & Viral capsid associated protein 39 & QHG11254.1 & - & - & - & 1 & - & - & - & - & - & - & - \\
\hline OrNV_gp106 & $v p 91$ & Viral capsid associated protein 91 & QHG11338.1 & 2 & 2 & - & 1 & - & 1 & 1 & 1 & 1 & - & - \\
\hline
\end{tabular}




\section{Figure legends}

Figure 1: Geographic locations of Coconut Rhinoceros beetles (O. rhinoceros) samples chronically infected with OrNV from Southeast Asia and the pacific islands (A). Host mitochondrial maximum likelihood phylogeny based on $O$. rhinoceros CoxI gene sequence. Four different haplotypes of $O$. rhinoceros, in three major haplotype clades (CRB-PNG, CRBS and CRB-G) were identified (B) Maximum likelihood phylogeny analysis of OrNV strains (C). Samples from Solomon Islands and Philippines are closely related to each other while individuals from PNG and Fiji are separated from them and located in another clade. Branch length indicates nucleotide substitutions per site, trees bootstrapped 1000 times. Host phylogenetic tree is midpoint rooted and

Figure 2: Genomic structural variants of different geographical populations of OrNV when compared to the newly sequenced reference genome of a Solomon Islands strain.

Figure 3: The number structural variants in coding and non-coding regions of different strains of OrNV.

Figure 4: Principal component analysis of OrNV samples from different regions based on the global viral gene expression profile.

Figure 5: Top 10 highly and poorly expressed OrNV genes in different geographical strains (A and B). Only 19 genes are differentially expressed among four OrNV strains (C).

Figure 6: OrNV is targeted by the host RNAi response and encodes a putative miRNA. The length distribution of the small RNA library (A). The length distribution of reads mapped to the OrNV genome which demonstrated a peak at $21 \mathrm{nt}(\mathbf{B})$ the red and blue bars represent reads mapped to negative and positive strands respectively. Noticeable hot spots were detected in several positions on the OrNV genome (C). The potential miRNA location is shown by an asterisk. OrNV-miR-1 hairpin loop structure (D). The small RNA read density mapped to 
different parts of potential OrNV precursor miRNA. Significant number of reads mapped to mature OrNV-miR-1-3p (E).

\section{Supplementary Files:}

Table S1: List of all identified structural variants on coding regions of OrNV among all different geographical strains.

Table S2: Differentially expressed viral genes among samples collected from the different populations.

Figure S1: The average coverage of RNAseq data mapped to the OrNV genome (Solomon Islands Strain). A consensus sequence of virus was generated for each sample.

Figure S2: OrNv-miR-1 binding sites on the genome of different geographical strains of OrNV. Venn diagram represents the number of common and strain specific binding sites (A). OrNv-miR-1-3p binding site on gp_027 exclusively in Fiji strains (B); OrNv-miR-1-5p binding site on gp_069 exclusively in PNG strains (C); OrNv-miR-1-5p binding site on gp_083 exclusively in Philippines strains (D); A common OrNv-miR-1-5p binding site on a non-coding part of genome in Fiji and PNG strains (E); A common OrNv-miR-1-3p binding site on gp_027 in Philippines, Solomon Islands and PNG strains (F); A common OrNv-miR-1-5p binding site on a non-coding part of genome in Philippines and Solomon Islands strains (G) 
bioRxiv preprint doi: https://doi.org/10.1101/2020.05.27.119867; this version posted May 27,2020 . The copyright holder for this preprint

(which was not certified by peer review) is the author/funder, who has granted bioRxiv a license to display the preprint in perpetuity. It is made available under aCC-BY-NC-ND 4.0 International license.

\section{Figure 1:}

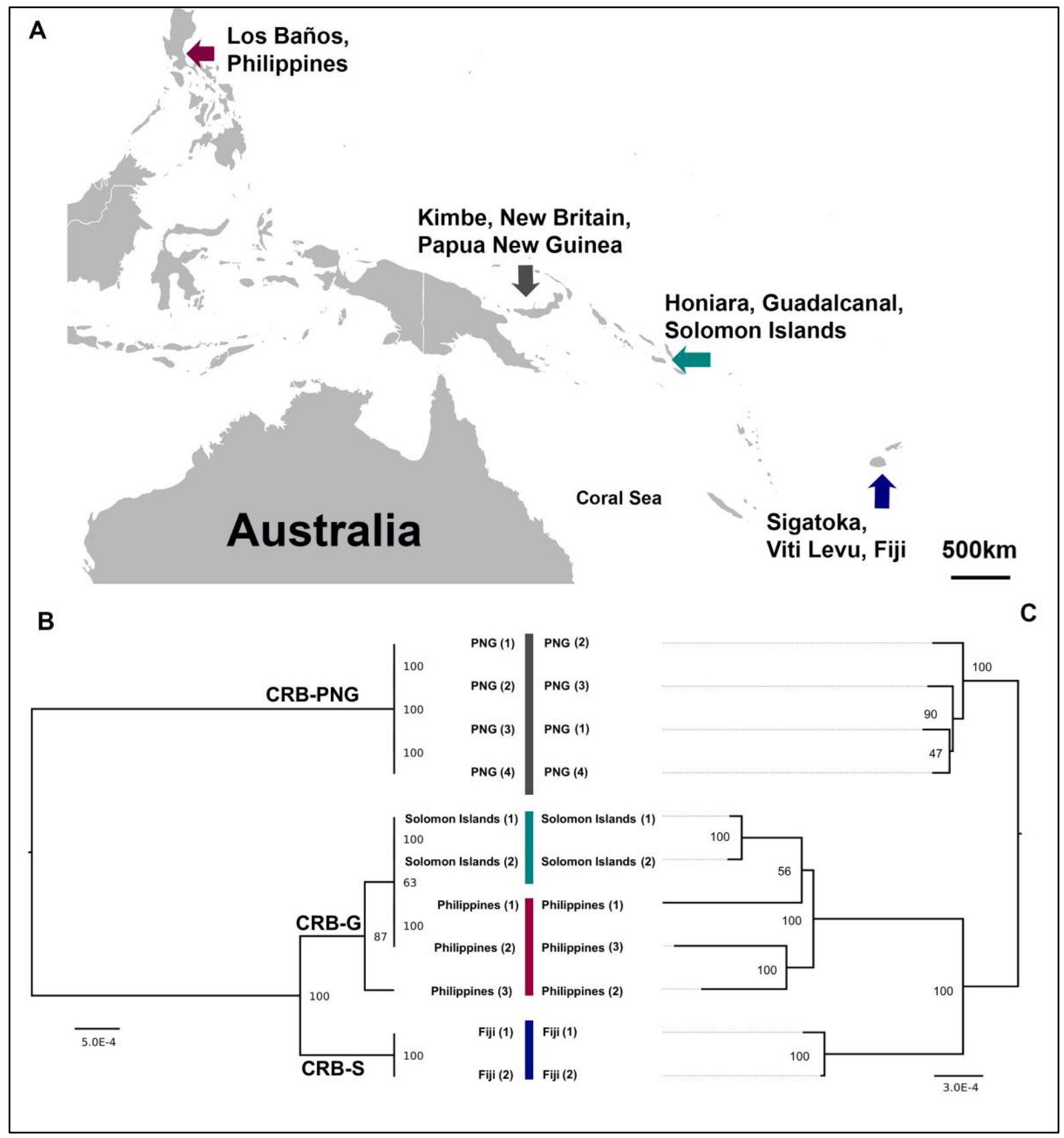


bioRxiv preprint doi: https://doi.org/10.1101/2020.05.27.119867; this version posted May 27, 2020. The copyright holder for this preprint

(which was not certified by peer review) is the author/funder, who has granted bioRxiv a license to display the preprint in perpetuity. It is made available under aCC-BY-NC-ND 4.0 International license.

\section{Figure 2:}
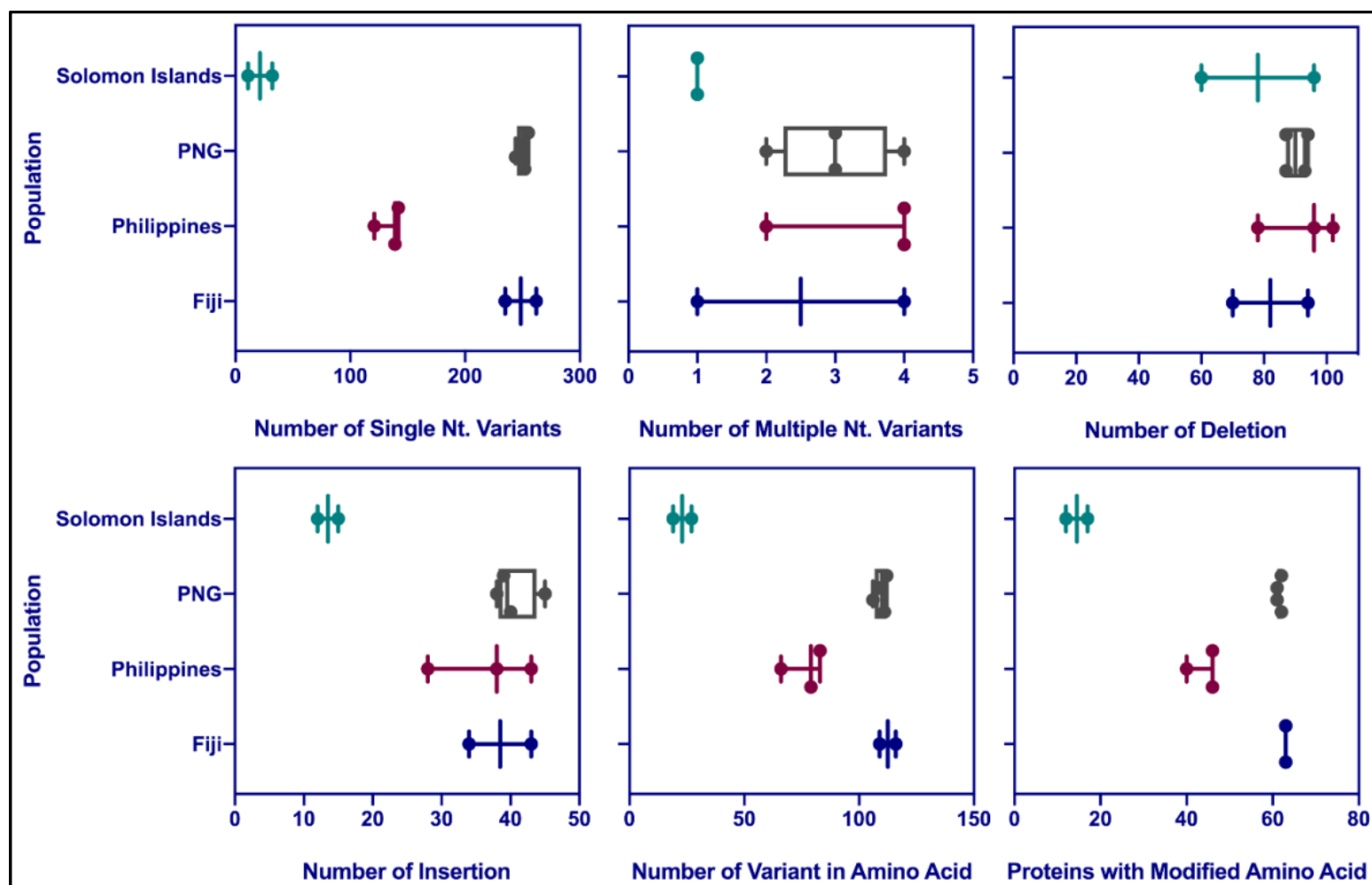

Number of Multiple Nt. Variants
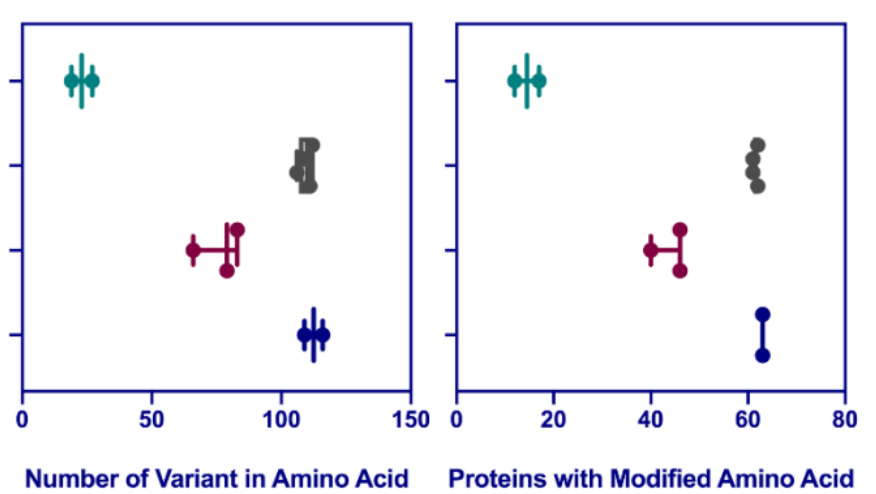


\section{Figure 3:}

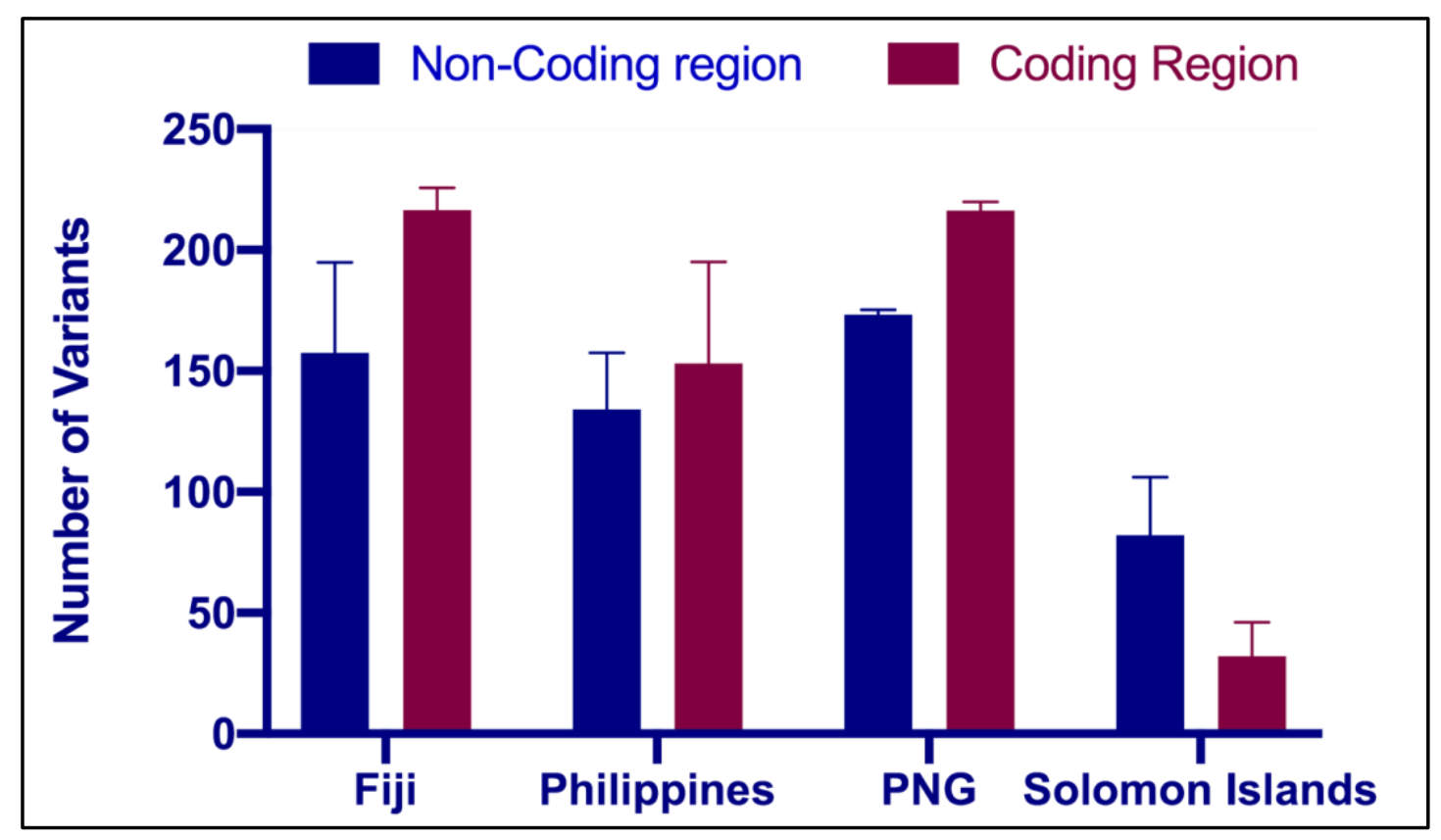


bioRxiv preprint doi: https://doi.org/10.1101/2020.05.27.119867; this version posted May 27,2020 . The copyright holder for this preprint (which was not certified by peer review) is the author/funder, who has granted bioRxiv a license to display the preprint in perpetuity. It is made available under aCC-BY-NC-ND 4.0 International license.

\section{Figure 4:}

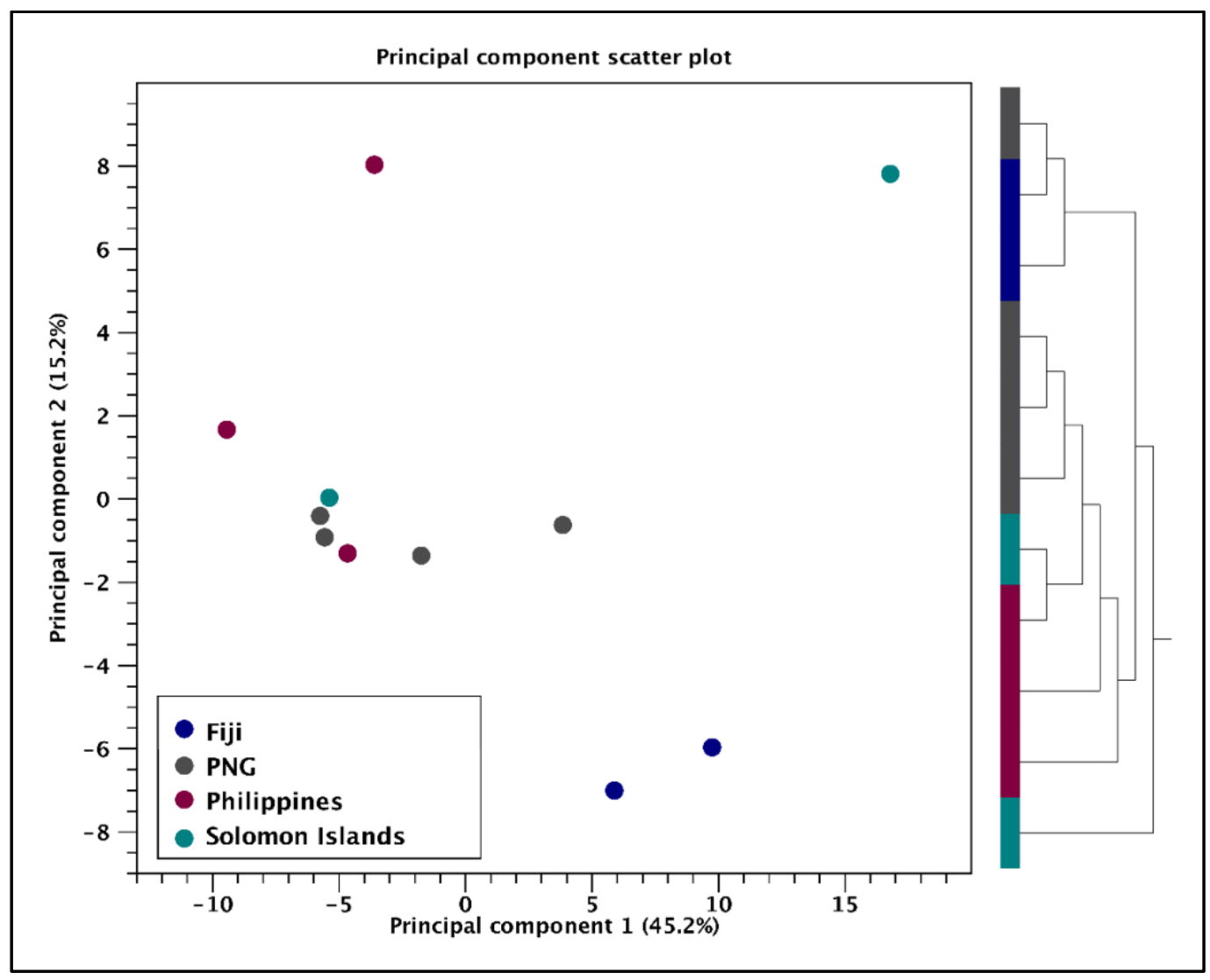


bioRxiv preprint doi: https://doi.org/10.1101/2020.05.27.119867; this version posted May 27, 2020. The copyright holder for this preprint (which was not certified by peer review) is the author/funder, who has granted bioRxiv a license to display the preprint in perpetuity. It is made available under aCC-BY-NC-ND 4.0 International license.

\section{Figure 5:}

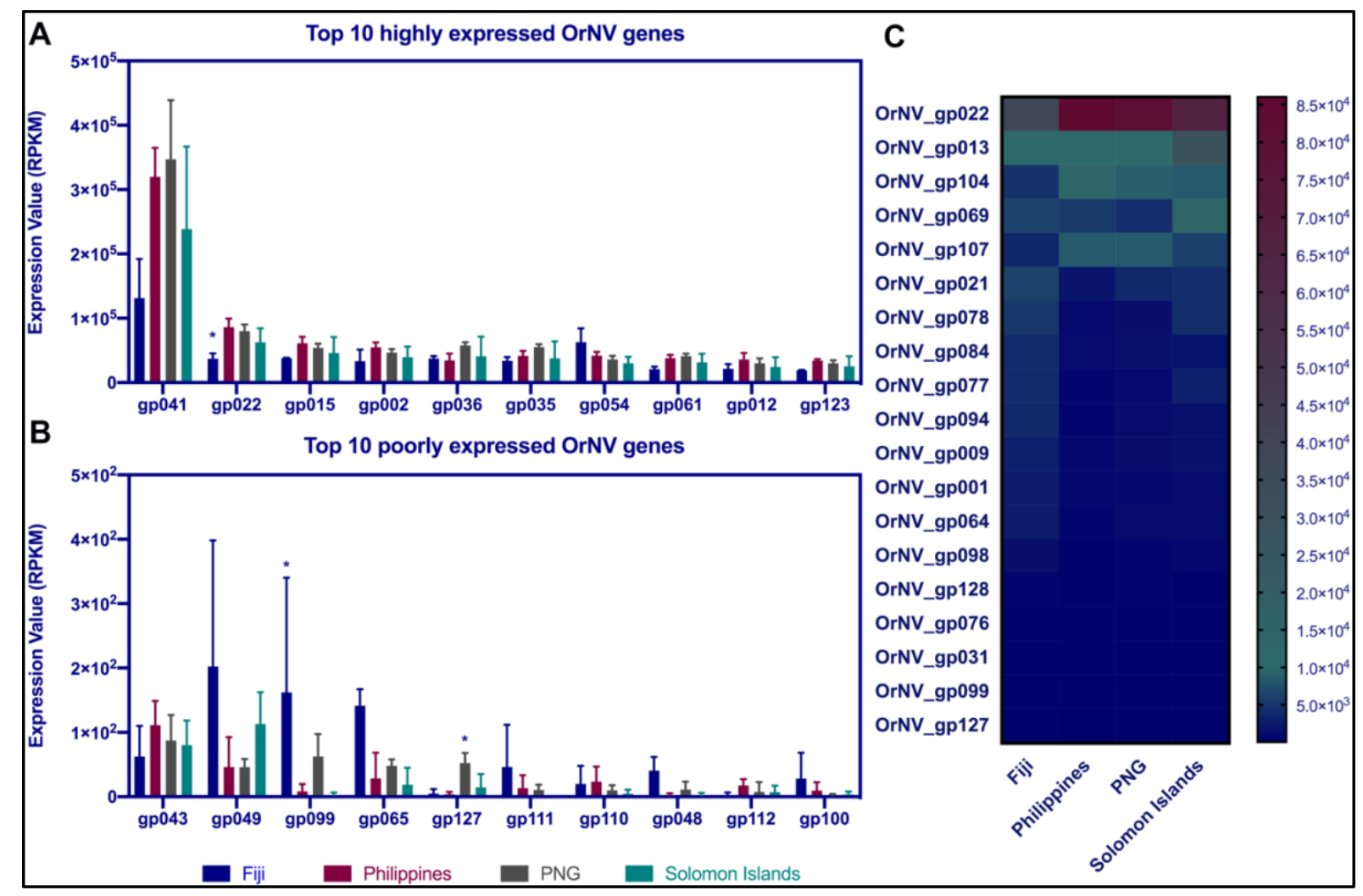


bioRxiv preprint doi: https://doi.org/10.1101/2020.05.27.119867; this version posted May 27, 2020. The copyright holder for this preprint

(which was not certified by peer review) is the author/funder, who has granted bioRxiv a license to display the preprint in perpetuity. It is made available under aCC-BY-NC-ND 4.0 International license.

\section{Figure 6:}

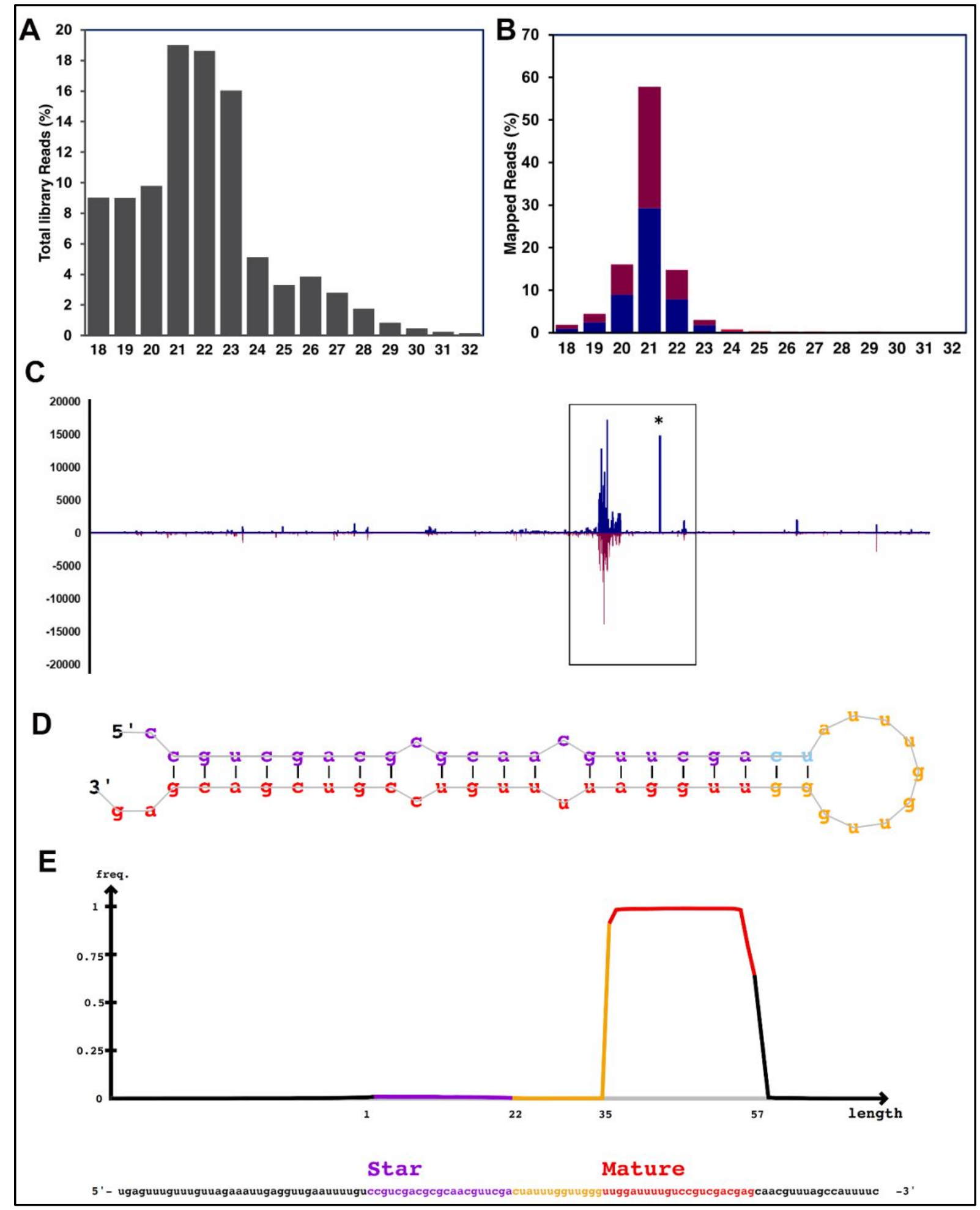


bioRxiv preprint doi: https://doi.org/10.1101/2020.05.27.119867; this version posted May 27, 2020. The copyright holder for this preprint

(which was not certified by peer review) is the author/funder, who has granted bioRxiv a license to display the preprint in perpetuity. It is made available under aCC-BY-NC-ND 4.0 International license.

\section{Figure S1:}

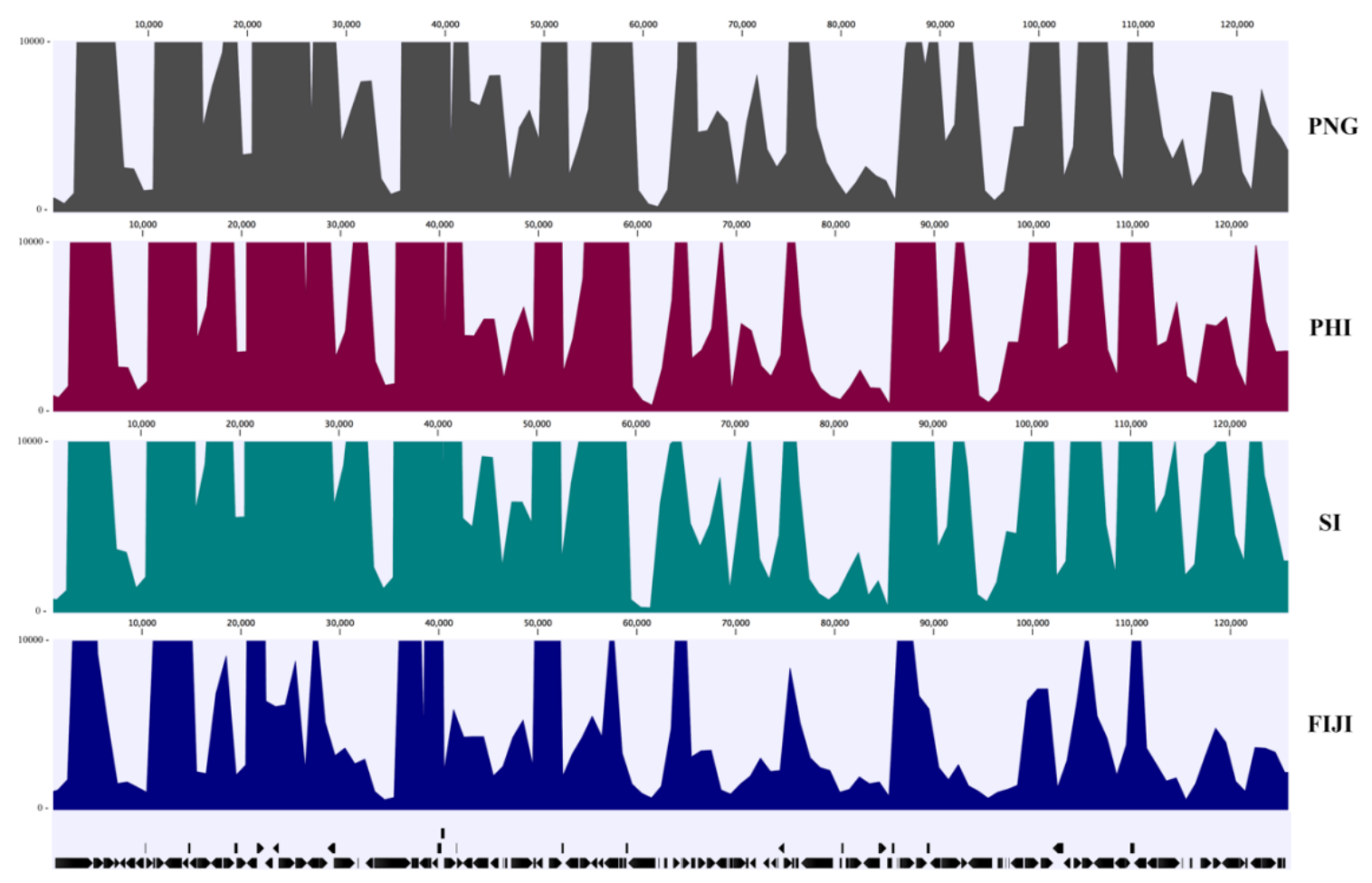


bioRxiv preprint doi: https://doi.org/10.1101/2020.05.27.119867; this version posted May 27, 2020. The copyright holder for this preprint (which was not certified by peer review) is the author/funder, who has granted bioRxiv a license to display the preprint in perpetuity. It is made available under aCC-BY-NC-ND 4.0 International license.

\section{Figure S2:}

A

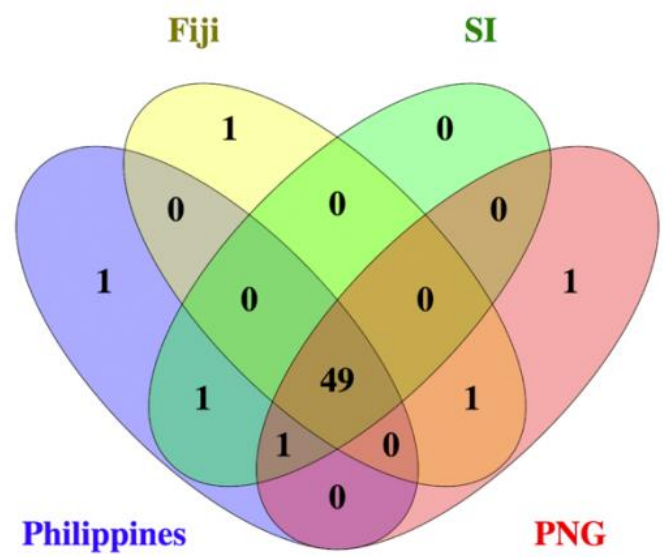

B

3' agctTGCAACGCGCAGCTGCC $5^{\prime}$ 5' atcATCATTTTG-GTCGACGg 3'

$-18.860001 \mathrm{kCal} / \mathrm{Mol}$

D

3' gCAGCTGCCTGTTTTAGGTt 5'

5. ttTCGGATTtTCAAATCCAg $3^{\prime}$

$-16.160000 \mathrm{kCal} / \mathrm{Mol}$

$\mathrm{F}$

3' agCTTGCA--ACGCGCAGCTGCC 5 ' 5' tagATCGTCATTTIGGTCGACGg 3. $-22.320000 \mathrm{kCal} / \mathrm{Mol}$
C

3' gcagctgcctgTTTTAGGTt 5'

5' tacattttctaAAAATCCAC 3'

$-8.230000 \mathrm{kCal} / \mathrm{Mol}$

E

3' gCAGCTGCCTGTTTTAGGTt 5 '

5' gctigaAaAAAAAAAATCCAa 3'

$-13.840000 \mathrm{kCal} / \mathrm{Mol}$

G

3' gcagctgccTGTTTTAGGTt 5 '

5' atgcttgaaAAAAAATCCAa $3^{\prime}$

$-13.960000 \mathrm{kCal} / \mathrm{Mol}$ 


\section{References}

1. Jehle JA: Nudiviruses: their Biology and Genetics. In: Insect Virology. Edited by Asgari S, Johnson KN: Caister Academic Press; 2010: 153-168.

2. Yang YT, Lee DY, Wang YJ, Hu JM, Li WH, Leu JH, Chang GD, Ke HM, Kang ST, Lin SS et al: The genome and occlusion bodies of marine Penaeus monodon nudivirus (PmNV, also known as MBV and PemoNPV) suggest that it should be assigned to a new nudivirus genus that is distinct from the terrestrial nudiviruses. BMC Genomics 2014, 15 :e628.

3. Wang YJ, Kleespies RG, Ramle MB, Jehle JA: Sequencing of the large dsDNA genome of Oryctes rhinoceros nudivirus using multiple displacement amplification of nanogram amounts of virus DNA. Journal of Virological Methods 2008, 152(1-2):106-108.

4. Etebari K, Filipovic I, Rasic G, Devine GJ, Tsatsia H, Furlong MJ: Complete genome sequence of Oryctes rhinoceros nudivirus isolated from the coconut rhinoceros beetle in Solomon Islands. Virus research 2020, 278:197864-197864.

5. Bedford GO: Biology and Management of Palm Dynastid Beetles: Recent Advances. In: Annual Review of Entomology, Vol 58. Edited by Berenbaum MR, vol. 58. Palo Alto: Annual Reviews; 2013: 353-372.

6. Huger AM: A virus disease of the Indian rhinoceros beetle, Oryctes rhinoceros (Linnaeus), caused by a new type of insect virus, Rhabdionvirus oryctes. Journal of Invertebrate Pathology 1966, 8(1):38-\&.

7. Marshall SDG, Moore A, Vaqalo M, Noble A, Jackson TA: A new haplotype of the coconut rhinoceros beetle, Oryctes rhinoceros, has escaped biological control by Oryctes rhinoceros nudivirus and is invading Pacific Islands. Journal of Invertebrate Pathology 2017, 149:127134.

8. Zelazny B, Alfiler AR, Lolong A: Possibility of resistance to a baculovirus in populations of the coconut rhinoceros beetle (Oryctes rhinoceros). FAO Plant Prot Bull 1989, 37:77-82.

9. Zelazny B, Alfiler AR: Ecology of baculovirus-infected and healthy adults of Oryctes rhinoceros (Coleoptera: Scarabaeidae) on coconut palms in the Philippines. Ecological Entomology 1991, 16(2):253-259.

10. Zelazny B, Lolong A, Pattang B: Oryctes rhinoceros (Coleoptera: Scarabaeidae) populations suppressed by a baculovirus. Journal of Invertebrate Pathology 1992, 59(1):6168.

11. Crawford AM, Zelazny B: Evolution in Oryctes baculovirus: rate and types of genomic change. Virology 1990, 174(1):294-298.

12. Moslim R, Kamarudin N, Ghani IA, Wahid MB, Jackson TA, Tey CC, Ahdly M: Molecular approaches in the assessment of Oryctes rhinoceros virus for the control of rhinoceros beetle in oil palm plantations. Journal of Oil Palm Research 2011, 23:1096-1109. 
13. Ramle M, Wahid MB, Norman K, Glare TR, Jackson TA: The incidence and use of Oryctes virus for control of rhinoceros beetle in oil palm plantations in Malaysia. Journal of Invertebrate Pathology 2005, 89(1):85-90.

14. Jackson TA, Crawford AM, Glare TR: Oryctes virus - Time for a new look at a useful biocontrol agent. Journal of Invertebrate Pathology 2005, 89(1):91-94.

15. Loiseau V, Herniou EA, Moreau Y, Leveque N, Meignin C, Daeffler L, Federici B, Cordaux R, Gilbert C: Wide spectrum and high frequency of genomic structural variation, including transposable elements, in large double-stranded DNA viruses. Virus evolution 2020, 6(1):vez060-vez060.

16. Chateigner A, Bezier A, Labrousse C, Jiolle D, Barbe V, Herniou EA: Ultra deep sequencing of a baculovirus population reveals widespread genomic variations. Viruses-Basel 2015, 7(7):3625-3646.

17. Theze J, Cabodevilla O, Palma L, Williams T, Caballero P, Herniou EA: Genomic diversity in European Spodoptera exigua multiple nucleopolyhedrovirus isolates. Journal of General Virology 2014, 95:2297-2309.

18. Shrestha A, Bao K, Chen Y-R, Chen W, Wang P, Fei Z, Blissard GW: Global analysis of Baculovirus Autographa californica Multiple Nucleopolyhedrovirus gene expression in the midgut of the lepidopteran host Trichoplusia ni. Journal of Virology 2018, $92(23)$.

19. Cory JS, Green BM, Paul RK, Hunter-Fujita F: Genotypic and phenotypic diversity of a baculovirus population within an individual insect host. Journal of Invertebrate Pathology 2005, 89(2):101-111.

20. Richards NK, Glare TR, Aloali'i I, Jackson TA: Primers for the detection of Oryctes virus from Scarabaeidae (Coleoptera). Molecular Ecology 1999, 8(9):1552-1553.

21. Folmer O, Black M, Hoeh W, Lutz R, Vrijenhoek R: DNA primers for amplification of mitochondrial cytochrome c oxidase subunit I from diverse metazoan invertebrates. Molecular Marine Biology and Biotechnology 1994, 3(5):294-299.

22. Mitchell AL, Attwood TK, Babbitt PC, Blum M, Bork P, Bridge A, Brown SD, Chang HY, ElGebali S, Fraser MI et al: InterPro in 2019: improving coverage, classification and access to protein sequence annotations. Nucleic Acids Research 2019, 47(D1):D351-D360.

23. Huerta-Cepas J, Szklarczyk D, Forslund K, Cook H, Heller D, Walter MC, Rattei T, Mende DR, Sunagawa S, Kuhn M et al: eggNOG 4.5: a hierarchical orthology framework with improved functional annotations for eukaryotic, prokaryotic and viral sequences. Nucleic Acids Research 2016, 44(D1):D286-D293.

24. Friedlander MR, Mackowiak SD, Li N, Chen W, Rajewsky N: miRDeep2 accurately identifies known and hundreds of novel microRNA genes in seven animal clades. Nucleic Acids Research 2012, 40(1):37-52. 
25. Enright AJ, John B, Gaul U, Tuschl T, Sander C, Marks DS: MicroRNA targets in Drosophila. Genome Biol 2004, 5(1):14.

26. Hill T, Unckless RL: The dynamic evolution of Drosophila innubila Nudivirus. Infection Genetics and Evolution 2018, 57:151-157.

27. Hill T, Thickless RL: Baculovirus molecular evolution via gene turnover and recurrent positive selection of key genes. Journal of Virology 2017, 91(22).

28. Ono C, Kamagata T, Taka H, Sahara K, Asano S, Bando H: Phenotypic grouping of 141 BmNPVs lacking viral gene sequences. Virus Research 2012, 165(2):197-206.

29. Ioannidis K, Swevers L, Iatrou K: Bombyx mori nucleopolyhedrovirus lef8 gene: effects of deletion and implications for gene transduction applications. Journal of General Virology 2016, 97:786-796.

30. Hitchman RB, Possee RD, King LA: High-Throughput Baculovirus Expression in Insect Cells. In: Recombinant Gene Expression: Reviews and Protocols, Third Edition. Edited by Lorence A, vol. 824; 2012: 609-627.

31. Cheng RL, Xi Y, Lou YH, Wang Z, Xu JY, Xu HJ, Zhang CX: Brown planthopper nudivirus DNA integrated in its host genome. Journal of Virology 2014, 88(10):5310-5318.

32. Burke GR: Common themes in three independently derived endogenous nudivirus elements in parasitoid wasps. Current Opinion in Insect Science 2019, 32:28-35.

33. Shi M, Lin XD, Tian JH, Chen LJ, Chen X, Li CX, Qin XC, Li J, Cao JP, Eden JS et al: Redefining the invertebrate RNA virosphere. Nature 2016, 540(7634):539-+.

34. Bazan JF, Fletterick RJ: Viral cysteine proteases are homologous to the trypsin-like family of serine proteases: structural and functional implications. Proceedings of the National Academy of Sciences of the United States of America 1988, 85(21):7872-7876.

35. Babe LM, Craik CS: Viral proteases: Evolution of diverse structural motifs to optimize function. Cell 1997, 91(4):427-430.

36. Palmer WH, Joosten J, Overheul GJ, Jansen PW, Vermeulen M, Obbard DJ, Van Rij RP: Induction and Suppression of NF-kappa B Signalling by a DNA Virus of Drosophila. Journal of Virology 2019, 93(3).

37. Hou DH, Kuang WH, Luo SJ, Zhang FH, Zhou FQ, Chen T, Zhang YF, Wang HL, Hu ZH, Deng F et al: Baculovirus ODV-E66 degrades larval peritrophic membrane to facilitate baculovirus oral infection. Virology 2019, 537:157-164.

38. Xiang XW, Chen L, Hu XL, Yu SF, Yang R, Wu XF: Autographa californica multiple nucleopolyhedrovirus odv-e66 is an essential gene required for oral infectivity. Virus Research 2011, 158(1-2):72-78.

39. Simon O, Williams T, Cerutti M, Caballero P, Lopez-Ferber M: Expression of a peroral infection factor determines pathogenicity and population structure in an insect virus. PLoS One 2013, 8(11):e0078834. 
40. Ono C, Kamagata T, Taka H, Sahara K, Asano S-i, Bando H: Phenotypic grouping of 141 BmNPVs lacking viral gene sequences. Virus Research 2012, 165(2):197-206.

41. Asgari S: microRNAs as Regulators of Insect Host-Pathogen Interactions and Immunity. In: Crop Protection. Edited by Smagghe G, vol. 55; 2018: 19-45.

42. Bronkhorst AW, van Rij RP: The long and short of antiviral defense: small RNA-based immunity in insects. Current Opinion in Virology 2014, 7:19-28.

43. Jayachandran B, Hussain M, Asgari S: RNA interference as a cellular defense mechanism against the DNA virus Baculovirus. Journal of Virology 2012, 86(24):13729-13734.

44. Sabin LR, Zheng Q, Thekkat P, Yang J, Hannon GJ, Gregory BD, Tudor M, Cherry S: Dicer2 processes diverse viral RNA species. PLoS One 2013, 8(2).

45. Parry R, Bishop C, De Hayr L, Asgari S: Density-dependent enhanced replication of a densovirus in Wolbachia-infected Aedes cells is associated with production of piRNAs and higher virus-derived siRNAs. Virology 2019, 528:89-100.

46. Hussain M, Asgari S: Functional analysis of a cellular microRNA in insect host-ascovirus interaction. Journal of Virology 2010, 84(1):612-620.

47. Wu YL, Wu CP, Liu CYY, Hsu PWC, Wu EC, Chao YC: A non-coding RNA of insect HzNV1 virus establishes latent viral infection through microRNA. Scientific Reports 2011, 1:e00060.

48. Hussain M, Taft RJ, Asgari S: An insect virus-encoded microRNA regulates viral replication. Journal of Virology 2008, 82(18):9164-9170.

49. Lewis SH, Quarles KA, Yang YJ, Tanguy M, Frezal L, Smith SA, Sharma PP, Cordaux R, Gilbert C, Giraud I et al: Pan-arthropod analysis reveals somatic piRNAs as an ancestral defence against transposable elements. Nature Ecology \& Evolution 2018, 2(1):174-181.

50. Webster CL, Waldron FM, Robertson S, Crowson D, Ferrari G, Quintana JF, Brouqui JM, Bayne EH, Longdon B, Buck AH et al: The discovery, distribution, and evolution of viruses associated with Drosophila melanogaster. PLoS Biology 2015, 13(7).

51. Kozomara A, Birgaoanu M, Griffiths-Jones S: miRBase: from microRNA sequences to function. Nucleic Acids Research 2019, 47(D1):D155-D162. 